\title{
A Receiving Antenna Allocation Scheme for Downlink MU-MIMO-OFDM Transmission
}

\author{
Tomoki Murakami ${ }^{1, *, \dagger}$, Yasushi Takatori ${ }^{1, \dagger}$ and Fumiaki Maehara ${ }^{2, \dagger}$ \\ 1 Access Network Service Systems Laboratories, NTT Corporation, Yokosuka 239-0847, Japan; \\ takatori.yasushi@lab.ntt.co.jp \\ 2 Graduate School of Fundamental Science and Engineering, Waseda University, Shinjuku 169-8555, Japan; \\ fumiaki_m@waseda.jp \\ * Correspondence: tomoki.murakami.nm@hco.ntt.co.jp; Tel.: +81-46-859-2057 \\ + These authors contributed equally to this work.
}

Received: 27 June 2018; Accepted: 23 July 2018; Published: 26 July 2018

\begin{abstract}
This paper proposes a novel receiving antenna allocation scheme for downlink multiuser multiple input multiple output orthogonal frequency division multiplexing (MU-MIMO-OFDM) transmission; an access point (AP) simultaneously transmits data frames to a combination of allocated receiving antennas on a subcarrier basis at each station (STA). The proposed scheme combines a limited channel state information (CSI) feedback sequence with a receiving antenna decision method. In the proposed scheme, each STA estimates the channel responses of all receiving antennas by using the training preamble transmitted from an $\mathrm{AP}$, and then feeds the channel response of the antenna with maximum norm back to the AP when the spatial correlation value between receiving antennas is higher than a threshold. Otherwise, each STA feeds full channel responses back to the AP. This scheme decreases the amount of CSI fed back while exploiting the spatial diversity gain, and the $\mathrm{AP}^{\prime}$ s computational complexity is also decreased regarding the antenna allocation. Moreover, the receiving antenna decision method eliminates the overhead to notify the allocated antenna information from the AP to each STA by simply comparing its own receiving antenna powers. We clarify the effectiveness of the proposed scheme in our computer simulations using channel responses measured in an indoor environment. The results show that the proposed scheme maintains the channel capacity of the downlink MU-MIMO-OFDM transmission while greatly reducing the overhead and computational complexity.
\end{abstract}

Keywords: downlink; multiuser multiple input multiple output (MU-MIMO); orthogonal frequency division multiplexing (OFDM); receiving antenna allocation; CSI feedback; wireless LAN

\section{Introduction}

To satisfy the growing demand for broadband wireless communication, it is necessary to enhance the channel capacity of wireless communication systems, such as mobile communication and wireless local area network (LAN) systems [1-4]. Downlink multiuser multiple input multiple output orthogonal frequency division multiplexing (MU-MIMO-OFDM) transmission, in which a transmitter simultaneously transmits individual data frames to multiple receivers, each with a few antennas, has been evaluated for wireless system standards, such as 3GPP long term evolution (LTE) advanced [5] and IEEE 802.11ac [6]. Furthermore, the downlink MU-MIMO-OFDM transmission with massive transmit antenna numbers is expected to greatly enhance the channel capacity $[7,8]$.

In the downlink MU-MIMO-OFDM transmission, inter-stream interference (ISI) is mitigated by pre-coding techniques, such as the linear processing approaches, including zero forcing (ZF) and block diagonalization (BD) $[9,10]$, and the non-linear processing approaches, such as Tomlinson-Harashima 
precoding and vector perturbation [11,12]. Conventional papers assume scenarios where the number of transmit antennas exceeds the total number of receiving antennas. In such scenarios, ISI can be greatly mitigated by using the degree of freedom $(\mathrm{DoF})$ of transmit antennas. However, if the number of receiving antennas at each STA increases, the DoF of transmit antennas will be insufficient. Thus, several studies have proposed coordinated transmitter-receiver (Tx-Rx) BD technique $[9,13]$, wherein one data stream is transmitted to each user, an initial set of received weights is assumed, and the optimal $\mathrm{Tx} / \mathrm{Rx}$ weights are alternatively recomputed until the solution converges to the one with minimum power and acceptable performance. This technique achieves higher channel capacity, but the computational complexity at the transmitter to calculate the $\mathrm{Tx} / \mathrm{Rx}$ weights and notification overhead created by the received weights becomes problematic.

As a more practical solution, the downlink MU-MIMO transmissions with receiving antenna allocation or selection schemes have been proposed for single carrier systems [14,15]. In these schemes, the transmitter simultaneously transmits data frames to an antenna combination, including the allocated antenna of each receiver. They attain the spatial diversity gain by exploiting the multiple antennas at each receiver, but the computational complexity at the transmitter to allocate the antenna combination with the maximum channel capacity from all combinations is high. In addition, it is necessary that each receiver must acquire information of the allocated receiving antenna before receiving the actual data frame. Since this overhead is proportional to the numbers of receivers, receiving antennas, and subcarriers, further degradation of the transmission efficiency occurs. For this problem, some papers have proposed techniques for reducing the computational complexity and overhead from a theoretical point of view [16-19]. However, there is no proposal that considers them in an integrated manner based on the existing wireless LAN systems.

This paper proposes a high-efficiency and low-complexity receiving antenna allocation scheme for the downlink MU-MIMO-OFDM transmission on IEEE 802.11ac [6]. In the assumed system, an AP simultaneously transmits data frames to the antenna combination allocated on a subcarrier basis at each STA. It achieves a higher channel capacity than using the common receiving antenna allocation for all subcarriers because of the spatial diversity gain. The proposed scheme uses the limited CSI feedback sequence based on [20] and the receiving antenna decision method based on [21]. First, in this sequence, each STA uses the training preamble transmitted from the AP to estimate channel responses as the CSI of all receiving antennas, and then feeds back to the AP just the response of the antenna, with the maximum norm assuming that the spatial correlation value between receiving antennas is higher than a threshold. Otherwise, each STA feeds full channel responses back to the AP. By using this approach, the amount of CSI feedback is decreased with only a small performance penalty. Moreover, the computational complexity incurred by the receiving antenna allocation at the AP is also decreased. The receiving antenna decision method also decreases the overhead associated with notifying each STA of the allocated antenna information; each STA estimates the allocated antenna by a power comparison of the receiving antennas at each STA. Moreover, the proposed method considers the antenna decision error to improve the accuracy of the antenna decision.

To clarify the effectiveness of the proposed scheme, we have measured the channel responses between AP and STAs in an indoor school environment and then analyzed the measured results. We have evaluated it by computer simulations using measured channel responses. The results show that the proposed scheme maintains the channel capacity of the downlink MU-MIMO-OFDM transmission with a greatly reduced overhead and computational complexity. Moreover, we show that the proposed method offers high accuracy in the antenna decision.

This paper is organized as follows: Section 2 presents a system model of the downlink MU-MIMO-OFDM transmission with the receiving antenna allocation; Section 3 describes the proposed scheme, including the limited CSI feedback sequence and the receiving antenna decision method; simulations using the measured results are shown in Section 4; and, finally, in Section 5, the paper is concluded. 


\section{System Model}

Figure 1 shows the system model of the downlink MU-MIMO-OFDM transmission wherein the AP communicates with $N$ STAs. We assume that the AP has $M$ antennas, and each STA has two antennas because most existing new smart phones, assumed as STA in this paper, are equipped with two antennas. $\boldsymbol{H}_{n}(k)=\left[\begin{array}{ll}\boldsymbol{h}_{1, n}(k)^{T} & \boldsymbol{h}_{2, n}(k)^{T}\end{array}\right]^{T} \in \mathbb{C}^{2 \times M}$ denotes the channel response of the $k$ th $(k=1, \ldots, K)$ subcarrier between the AP and STA- $n . A^{T}$ is the transpose operator of $\boldsymbol{A} . \boldsymbol{h}_{1, n}(k)$ and $\boldsymbol{h}_{2, n}(k)$ are the channel responses between AP and the first and second antennas of STA- $n$, respectively. Moreover, this paper assumes the downlink MU-MIMO-OFDM transmission with the receiving antenna allocation per subcarrier, so the AP simultaneously transmits individual data frames to the antenna combination, including the allocated receiving antennas at STAs to obtain the spatial diversity gain. On the other hand, each STA receives its own data via its allocated antenna. Although the STA can use the signal from the non-allocated antenna, this signal includes a large amount of ISI because the non-allocated antenna is not supported by the pre-coding for ISI calculation at the AP. Therefore, this paper focuses on the reception via the allocated antenna. The channel response between the AP and STA- $n$ after applying the receiving antenna allocation yields, $\hat{h}_{n}(k)$. In the downlink MU-MIMO-OFDM transmission, the AP acquires channel responses to calculate the pre-coding weights before actual data transmission. Given the transmit signal, $x_{n}(k)$, the received signal of the $k$ th subcarrier at the STA- $n$, $y_{n}(k)$, is expressed as:

$$
y_{n}(k)=\sqrt{P_{n}} \hat{\boldsymbol{h}}_{n}(k) \boldsymbol{w}_{n}(k) x_{n}(k)+\sum_{l=1, l \neq n}^{N} \sqrt{P_{l}} \hat{\boldsymbol{h}}_{n}(k) \boldsymbol{w}_{l}(k) x_{l}(k)+z_{n}(k),
$$

where $P_{n}$ is the transmit power for STA-n, $w_{n}(k) \in \mathbb{C}^{M \times 1}$, is the pre-coding weight of the $k$ th subcarrier for STA- $n$, and $z_{n}(k)$ is the additive white Gaussian noise vector of the $k$ th subcarrier with a variance of $\sigma^{2}$. It is assumed that equal power is allocated to each STA, $P=P_{n}$, to prevent unfairness in terms of the transmission quality between STAs. For practical use, the pre-coding weight assumed in this paper is calculated by channel inversion based on the ZF [9,10], a low complexity weight calculation method given by:

$$
\begin{aligned}
& \boldsymbol{W}(k)=\left[\begin{array}{lllll}
\frac{w_{1}(k)}{\left\|\boldsymbol{w}_{1}(k)\right\|_{F}} & \cdots & \frac{w_{n}(k)}{\left\|\boldsymbol{w}_{n}(k)\right\|_{F}} & \cdots & \frac{w_{N}(k)}{\left\|\boldsymbol{w}_{N}(k)\right\|_{F}}
\end{array}\right], \\
& {\left[\begin{array}{lllll}
\frac{w_{1}(k)}{\left\|\boldsymbol{w}_{1}(k)\right\|_{F}} & \cdots & \frac{\boldsymbol{w}_{n}(k)}{\left\|\boldsymbol{w}_{n}(k)\right\|_{F}} & \cdots & \frac{\boldsymbol{w}_{N}(k)}{\left\|\boldsymbol{w}_{N}(k)\right\|_{F}}
\end{array}\right]=\hat{\boldsymbol{H}}(k)^{H}\left(\hat{\boldsymbol{H}}(k) \hat{\boldsymbol{H}}(k)^{H}\right)^{-1},}
\end{aligned}
$$

where $\|\boldsymbol{A}\|_{F}$ denotes the Frobenius norm of $\boldsymbol{A}$, and $\hat{\boldsymbol{H}}(k)$ is expressed as:

$$
\hat{\boldsymbol{H}}(k)=\left[\begin{array}{lllll}
\hat{\boldsymbol{h}}_{1}(k)^{T} & \ldots & \hat{\boldsymbol{h}}_{n}(k)^{T} & \ldots & \hat{\boldsymbol{h}}_{N}(k)^{T}
\end{array}\right]^{T} .
$$

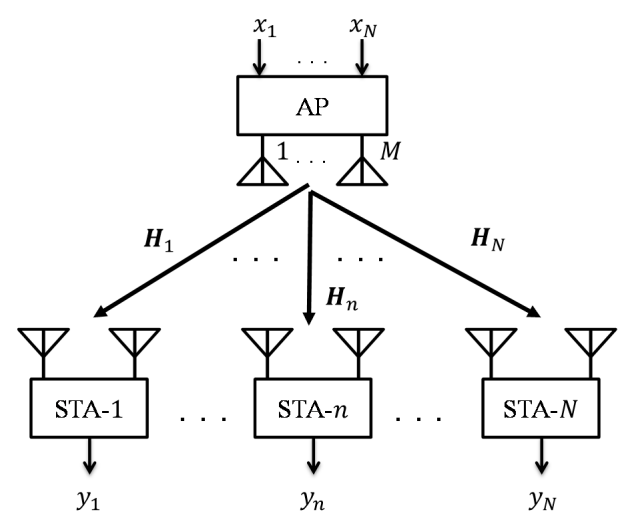

Figure 1. System model in which the access point (AP), using $M$ antennas, communicates with $N$ stations (STAs) using two antennas on the downlink multiuser multiple input multiple output orthogonal frequency division multiplexing (MU-MIMO-OFDM) transmission. 
By using the pre-coding weight with Equation (2), the downlink MU-MIMO-OFDM transmission to the antenna combination is realized. To evaluate the basic performance of the proposed scheme, this paper assumes the greedy algorithm; it allocates the antenna combination, $s(k)$, for the $k$ th subcarrier that maximizes the channel capacity from all antenna combinations, $S$. The channel capacity of the $k$ th subcarrier and the $s(k)$ th antenna combination at STA- $n, C_{n}(k, s(k))$ is calculated by:

$$
C_{n}(k, s(k))=\log _{2}\left(1+\operatorname{SINR}_{n}(k, s(k))\right),
$$

where $\operatorname{SINR}_{n}(k, s(k))$ is the signal to interference plus noise power ratio (SINR) given by:

$$
\operatorname{SINR}_{n}(k, s(k))=\frac{\left|\sqrt{P} \hat{\boldsymbol{h}}_{n, s(k)}(k) \boldsymbol{w}_{n, s(k)}(k)\right|^{2}}{\sigma^{2}+\sum_{l=1, l \neq n}^{N}\left|\sqrt{P} \hat{\boldsymbol{h}}_{n, s(k)}(k) \boldsymbol{w}_{l, s(k)}(k)\right|^{2}} .
$$

Finally, the allocated antenna combination with a maximum channel capacity, $s_{\max }(k)$, is calculated by:

$$
S_{\text {max }}(k)=\underset{s(k)}{\operatorname{argmax}}\left(\sum_{n=1}^{N} C_{n}(k, s(k))\right) .
$$

\section{Proposed Scheme}

This section introduces our high-efficiency and low-complexity receiving antenna allocation scheme for the downlink MU-MIMO-OFDM transmission based on IEEE 802.11ac [6]. The proposed scheme combines the limited CSI feedback sequence based on [20] and receiving antenna decision method based on [21].

\subsection{Limited CSI Feedback Sequence}

Figure 2 shows the proposed frame sequence, including limited CSI feedback, MU-MIMO-OFDM transmission, and acknowledgement subsequences. It is assumed that the signaling complies with the frame formats of IEEE802.11ac [6]. In the limited CSI feedback subsequence, the AP first broadcasts a null data packet announcement (NDPA) and NDP frames to STAs for estimating channel responses on a subcarrier basis; each STA estimates channel responses from the received NDP frame and calculates the spatial correlation value between receiving antennas for each subcarrier, $\rho_{n}(k)$, as:

$$
\rho_{n}(k)=\frac{\left|\boldsymbol{h}_{1, n}(k) \cdot \boldsymbol{h}_{2, n}(k)^{H}\right|}{\left\|\boldsymbol{h}_{1, n}(k)\right\|\left\|_{F}|| \boldsymbol{h}_{2, n}(k)\right\|_{F}} .
$$

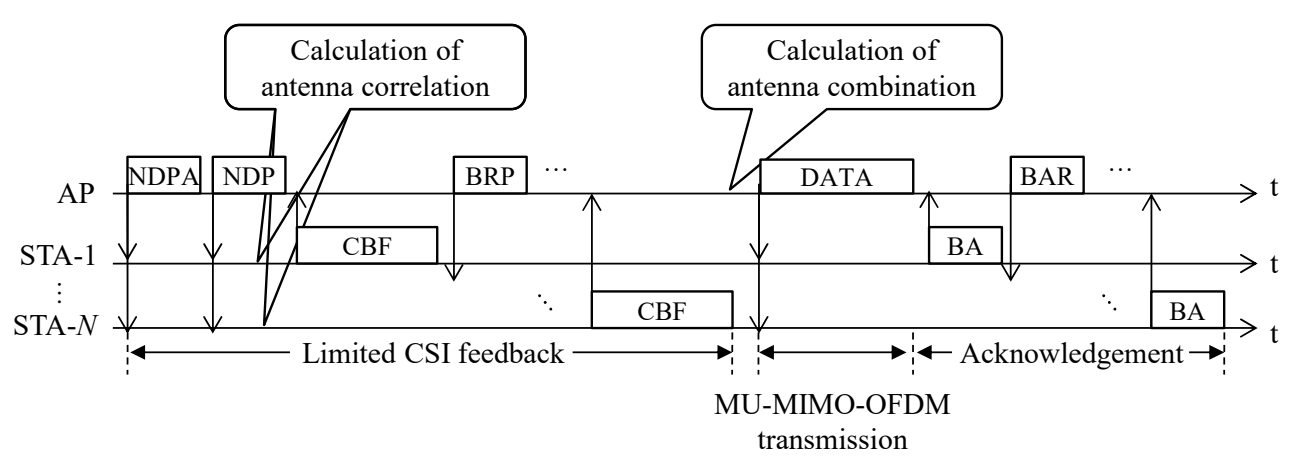

Figure 2. Proposed frame sequence, including limited channel state information (CSI) feedback, MU-MIMO-OFDM transmission, and acknowledgement subsequences based on frame formats of IEEE802.11ac [6]. 
Then, each STA feeds back just the channel response of the antenna with maximum norm to the AP via a compressed beamforming frame $(\mathrm{CBF})$ when the spatial correlation value between the receiving antennas exceeds a threshold, $\rho_{t h}$. Note that this threshold is a parameter that determines the tradeoff between spatial diversity gain and CSI feedback reduction. The CSI feedback quantity is up to half that of the conventional value. Moreover, it is generally known that the channel capacity in MIMO transmission depends on the received signal power and spatial correlation. Since it can be expected that using the antenna with a higher received signal power improves the channel capacity, this paper selects the channel response of the antenna with maximum norm. Otherwise, each STA feeds full channel responses back to the AP. This paper assumes unitary matrix compression feedback, so the channel response is sent in the form of angles representing compressed beamforming feedback matrices, $V$, per subcarriers, as supported by IEEE802.11ac [6]. The AP lets other STAs feed the channel response back to the AP in response of the beamforming report poll (BRP) frame. After receiving the channel responses, the AP calculates its pre-coding weights and allocated antenna combination using Equations (2)-(7). In the MU-MIMO-OFDM transmission subsequence, the AP then transmits the data frames to multiple STAs for the downlink MU-MIMO-OFDM transmission. Finally, in the acknowledgement subsequence, the AP and STA check for the existence of errors by block acknowledgment (BA) and BA request (BAR).

\subsection{Receiving Antenna Decision Method}

Both the coordinated Tx-Rx BD techniques $[9,13]$ and DL-MU-MIMO transmissions with receiving antenna allocation/selection schemes [14,15] must require information of the receiver weights or allocated receiving antennas before receiving actual data frames, so the transmission efficiency, which is the ratio of time spent transmitting actual data to total time, is degraded. To reduce this overhead, the proposed method determines the receiving antenna by comparing the received powers of each antenna at STA using a few OFDM signals. In detail, each STA determines the receiving antenna with minimum received power as calculated from OFDM signals among all antennas using:

$$
\left\{\begin{array}{l}
\hat{\boldsymbol{h}}_{n}(k)=\boldsymbol{h}_{1, n}(k) \text { if }\left|y_{1, n}(k)\right| \leq\left|y_{2, n}(k)\right| \\
\hat{\boldsymbol{h}}_{n}(k)=\boldsymbol{h}_{2, n}(k) \text { if }\left|y_{1, n}(k)\right|>\left|y_{2, n}(k)\right|
\end{array},\right.
$$

where $y_{1, n}(k)$ and $y_{2, n}(k)$ are the received signals of the first and second antennas, respectively. Moreover, for improving the accuracy of the receiving antenna decision, we assume that the pre-coding weight, $w \prime(k)$, which defines the right singular vector corresponding to the minimum eigenvalue, is given by:

$$
\begin{gathered}
\boldsymbol{w} \prime(k)=\hat{\boldsymbol{v}}_{N}(k), \\
\hat{\boldsymbol{H}}(k)=\hat{\boldsymbol{U}}(k) \hat{\boldsymbol{\Sigma}}(k) \hat{\boldsymbol{V}}(k)^{H}, \\
\hat{\boldsymbol{V}}(k)=\left[\begin{array}{lll}
\hat{\boldsymbol{v}}_{1}(k) & \cdots & \hat{\boldsymbol{v}}_{N}(k)
\end{array}\right],
\end{gathered}
$$

where $A^{H}$ denotes the Hermitian transpose operator of $A, \hat{U}(k) \in \mathbb{C}^{N \times N}$ is the matrix combined from the left singular vectors of the $k$ th subcarrier, $\hat{\Sigma}(k) \in \mathbb{C}^{N \times N}$ is the diagonal matrix of the $k$ th subcarrier in which the diagonal elements represent the square roots of the eigenvalues, and $\hat{V}(k) \in \mathbb{C}^{N \times N}$ is the matrix combined from the right singular vectors of the $k$ th subcarrier.

Actual propagation environments exhibit frequency selective fading, shadowing, transmit beamforming, etc., so the STAs may err in determining the receiving antenna. To further improve the accuracy of the antenna decision, this paper applies a receiving antenna allocation scheme that applies 
the receiving antenna decision method. In this proposal, the AP calculates the SINR to estimate the receiving antenna per subcarrier at each STA, given by:

$$
\left\{\begin{aligned}
\operatorname{SINR}_{1, n}(k, s(k)) & =\frac{\left|\sqrt{P} \hat{\boldsymbol{h}}_{1, n, s(k)}(k) \boldsymbol{w}_{h, s(k)}(k)\right|^{2}}{\sigma^{2}+\sum_{l=1, l, n}^{N}\left|\sqrt{P} \hat{\boldsymbol{h}}_{n, s(k)}(k) \boldsymbol{w}_{l, s(k)}(k)\right|^{2}} \\
\operatorname{SINR}_{n}(k, s(k)) & =\frac{\left|\sqrt{P} \hat{\boldsymbol{h}}_{h, s(k)}(k) \boldsymbol{w}_{n, s(k)}(k)\right|^{2}}{\sigma^{2}+\sum_{l=1, l \neq n}^{N}\left|\sqrt{P} \hat{\boldsymbol{h}}_{h, s(k)}(k) \boldsymbol{w}_{l, s(k)}(k)\right|^{2}}
\end{aligned}\right.
$$

where $\operatorname{SINR}_{1, n}(k, s(k))$ and $\operatorname{SINR}_{2, n}(k, s(k))$ are the SINR values of the first and second antennas, respectively. The AP estimates the receiving antenna decision error at STA by using Equation (13). In addition, this process is performed only for the STAs that fed both channel responses back to the AP. Finally, the antenna combination that maximizes the channel capacity is calculated by Equations (6) and (7) from candidates without the antennas that may cause errors in the antenna decision.

We present an example frame format for the proposed method that suits IEEE 802.11ac. This frame is used by the STAs that fed back both channel responses, while STAs that fed back the channel response of a single antenna uses a general receiving method with a selected antenna. Figure $3 a, b$ show the example frame format with proposed/general pre-cording regions and the receiving process with the concept of the proposed antenna decision method, respectively. The frame format of Figure 3a consists of a legacy short training field (L-STF), a legacy long training field (L-LTF), and a legacy signal field (L-SIG) for backward compatibility, and a very high throughput signal field A (VHT-SIG-A), a VHT-STF, VHT-LTFs, a VHT-SIG-B, and data signals. When the downlink MU-MIMO-OFDM transmission is used, pre-coding is performed for the signal region from VHT-STF to the end of the data signals. On the other hand, pre-coding of the proposed method is performed for the signal region from L-STF to VHT-SIG-A. When a signal is received by the STA in Figure 3b, the STA calculates the received VHT-SIG-A and decides the allocated antenna using the powers calculated for the signal region from L-STF to the minimum received power among all the antennas. At the same time, the STA acquires information (modulation and coding rate scheme, frame length, and so on) necessary for demodulation from the signal captured by the non-allocated antenna. The STA then demodulates the signal after VHT-STF by using the decided antenna. By using the above frame format and receiving process, the proposed method can be realized.

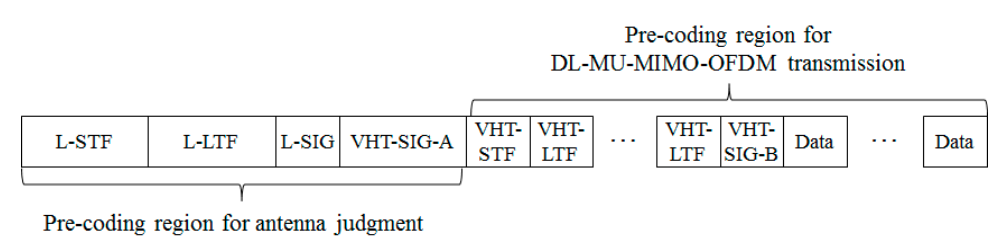

(a)

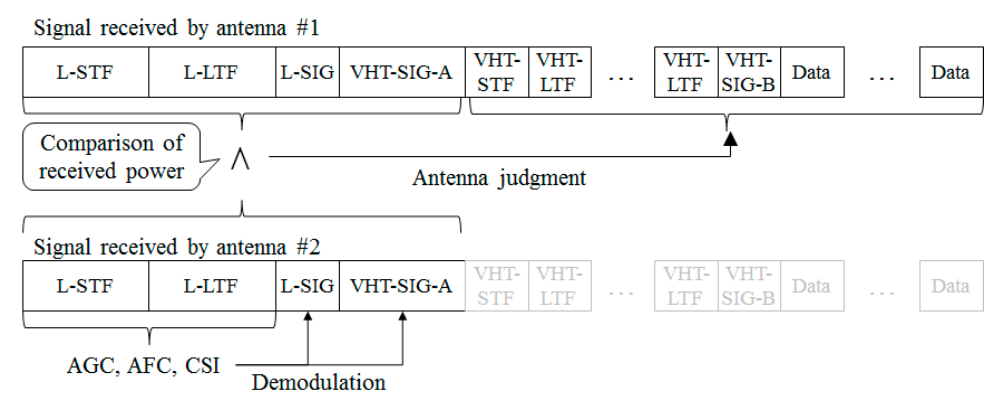

(b)

Figure 3. Frame format and receiving process for IEEE 802.11ac. (a) Example frame format with the proposed pre-cording region and general pre-coding region; and $(\mathbf{b})$ the receiving process with the concept of the proposed antenna decision method. 


\section{Performance Evaluation}

In this paper, we have evaluated the proposed scheme by computer simulations using channel responses measured in an actual environment to clarify the potential of it.

\subsection{Experimental Measurements of Channel Responses}

Simulated or measured channel responses can be used to evaluate the proposed scheme. Since we consider that it is commercially important to show the effect in practical, existing usage scenarios, we used measured MIMO-OFDM channel responses captured between the AP and STAs in an indoor static line-of-sight (LOS) school environment, as shown in Figure 4. AP sets were at the front, as shown in Figure 4. We measured MIMO-OFDM channel responses at 22 STA locations. The measurement conditions are shown in Table 1. In the measurement, the receiver (Digital Signal Analyzer DSA90804A [22]) installed at the STA location captured beacon signals, including short and long preambles, transmitted from the AP, and calculated channel responses from the captured signals. The measurements were conducted at $5.18 \mathrm{GHz}$. The bandwidth and the number of subcarriers were $20 \mathrm{MHz}$ and 52, respectively. The transmit power was $13 \mathrm{dBm}$. The AP had four dipole antennas with an element spacing of 0.5 wavelengths. The STA had two dipole antennas with an element spacing of 0.5 wavelengths. The heights of the AP and STA were $1 \mathrm{~m}$ above the floor. The measured channel responses were normalized and yielded an average signal to noise ratio (SNR) of $20 \mathrm{~dB}$ because of the basic evaluation in terms of the spatial correlation.

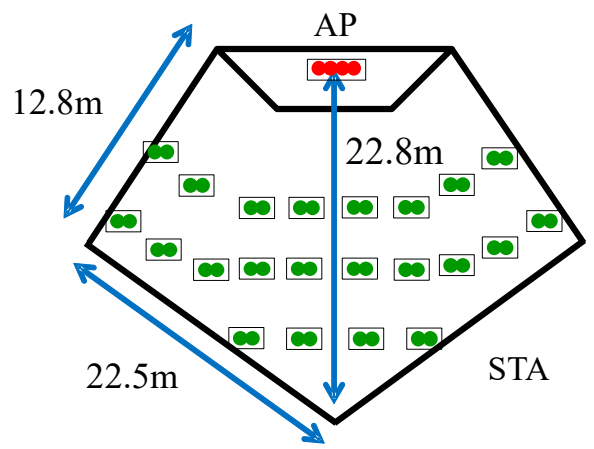

(a)

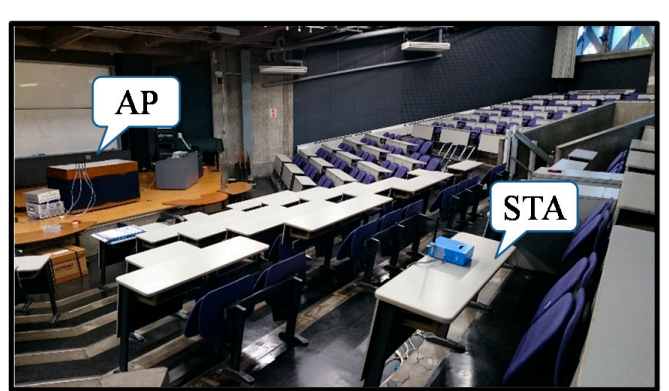

(b)

Figure 4. Experimental environments. (a) Room layout; and (b) photograph of room.

Table 1. Experimental conditions.

\begin{tabular}{cc}
\hline Parameter & Value \\
\hline Center frequency & $5.18 \mathrm{GHz}$ \\
Bandwidth (Subcarrier number) & $20 \mathrm{MHz}(52)$ \\
Transmit power & $13 \mathrm{dBm}$ \\
Number of AP antennas & 4 \\
Number of STA antennas & 2 \\
Transmit/Receiving antennas (Gain) & Dipole (2 dBi) \\
\hline
\end{tabular}

\subsection{Validity of DL-MU-MIMO-OFDM Transmission Using Receiving Antenna Allocation}

We show the potential channel capacity of the downlink MU-MIMO-OFDM transmission with the receiving antenna allocation per subcarrier. Figure 5 shows the cumulative distribution function (CDF) of the total channel capacities per subcarrier by using the coordinated Tx-Rx BD technique [13] and MU-MIMO-OFDM transmission with antenna allocation [14,15]. In the coordinated Tx-Rx BD technique, pre-coding and receiver weights were updated 10 times. Antenna allocation was performed randomly or in optimal manner. Optimum means to use the antenna that offers the maximum SINR among all antenna combinations. The result of the single user MIMO (SU-MIMO) with time division 
multiplexing access (TDMA) is also plotted as reference data. These results show the coordinated Tx-Rx BD technique achieves a higher channel capacity than the other methods. This is because the DoF of all receiver antennas can be used. However, as shown in the Introduction, its demerit is a higher computational complexity for the receiver weight multiplication and a greater overhead for notification of the quantized receiver weight. The channel capacity of the downlink MU-MIMO-OFDM transmission with the receiving antenna allocation per subcarrier is slightly degraded than that of the coordinated Tx-Rx BD technique even though each STA uses only one antenna. This is because the spatial diversity gain is more effectively attained by allocating antennas on a per subcarrier basis. Therefore, the proposed method is effective when there are massive numbers of wireless devices to service.

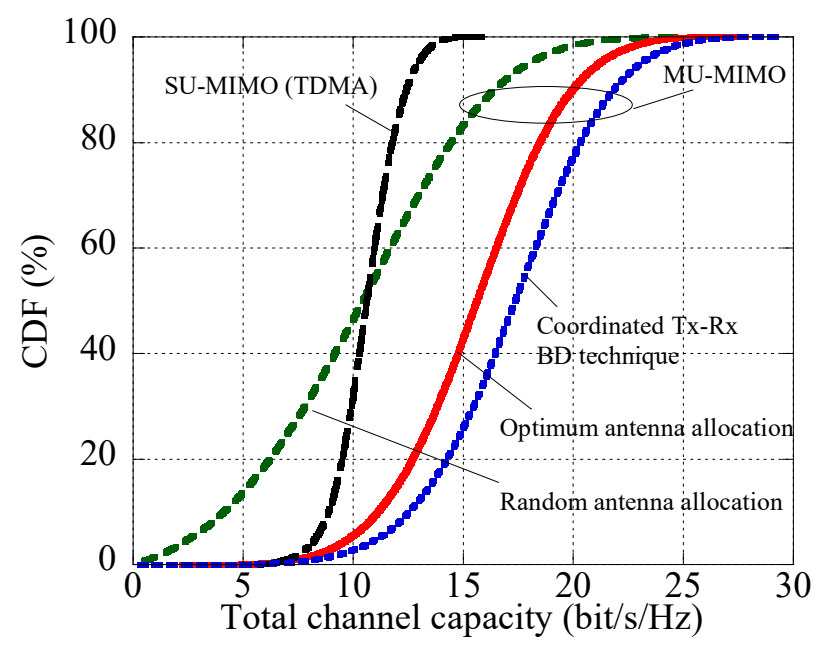

Figure 5. Cumulative distribution function (CDF) of the total channel capacities of the coordinated transmitter-receiver (Tx-Rx) BD technique, MU-MIMO-OFDM transmission with optimum/random antenna allocations, and single user MIMO (SU-MIMO) transmission on the time division multiplexing access (TDMA).

\subsection{Analysis of Limited CSI Feedback Sequence}

Figure 6 plots the averages of the total channel capacity per subcarrier on the downlink MU-MIMO-OFDM transmission and the reduction rate by using the proposed frame sequence versus the threshold value, $\rho_{t h}$. Reduction rate means the rate between the average amount of CSI feedback by using the proposed scheme and the amount of CSI feedback based on two antennas. First, the total channel capacity increases with the threshold value. This is because the number of antenna combinations also increases. As expected, the overhead reduction rate falls. However, even low threshold values greatly reduce the number of antenna combinations and the CSI feedback overhead. For example, the reduction rates are $0.59\left(\rho_{t h}=0.5\right), 0.62\left(\rho_{t h}=0.6\right), 0.7 \%\left(\rho_{t h}=0.7\right)$, $0.78\left(\rho_{t h}=0.8\right)$, and $0.88\left(\rho_{t h}=0.9\right)$ in our indoor school environment. From these plots, the channel capacity and overhead reduction rate have a trade-off relationship, so the threshold value should be adjusted to suit the use case. Furthermore, system performance, including the overhead penalty of the CSI feedback, can be evaluated by defining the durations of the data and overhead frames. For example, assuming that the durations of data and overhead frames are the same, we found that the system performance of the proposed scheme with threshold optimization is about $30 \%$ higher than that of the conventional scheme using random antenna allocation. 


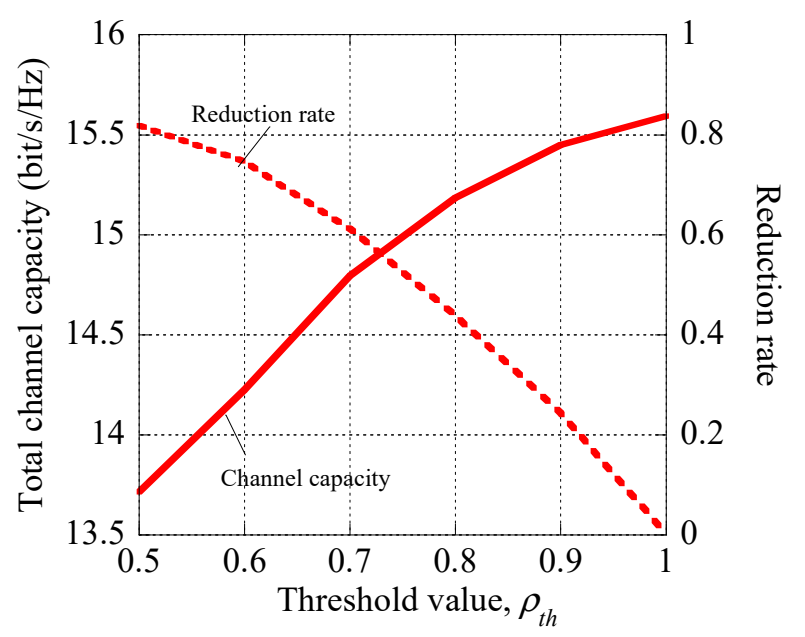

Figure 6. Total channel capacity and reduction rate vs. threshold value.

Figure 7 plots the CDF of the total channel capacities for the proposal using three $\rho_{\text {th }}$ values, $(0.5,0.7$, and 0.9$)$, and random/optimum antenna allocations. Optimum means to use the antenna that offers the maximum SINR among all antenna combinations. This figure showed that the proposed scheme offers a higher channel capacity than random antenna allocation. Moreover, the offsets from the channel capacity with optimum antenna allocation are less than $20 \%$ at a CDF value of $50 \%$.

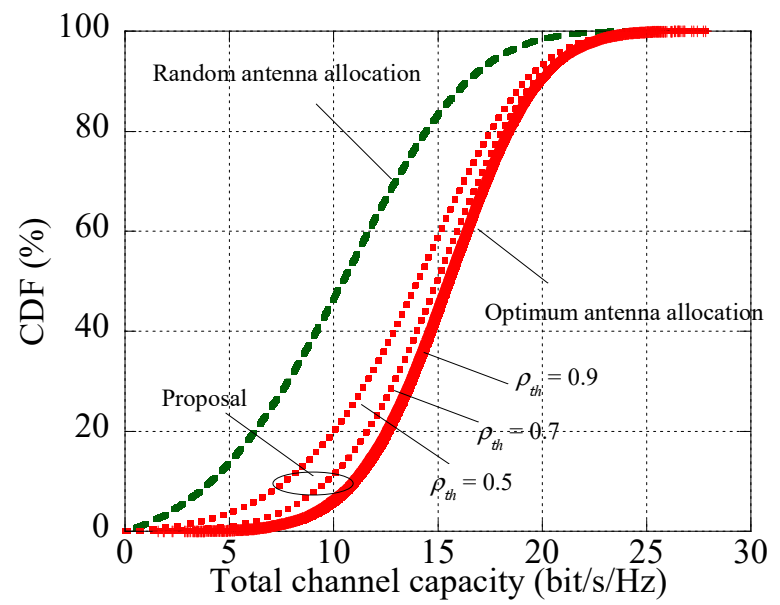

Figure 7. CDF of the total channel capacities of the downlink MU-MIMO-OFDM transmission with the proposal and random/optimum antenna allocations.

\subsection{Analysis of Receiving Antenna Decision Method}

Figure 8 plots the overhead time versus the number of STAs. In the conventional downlink MU-MIMO-OFDM transmission assumed in this paper, each STA needs to acquire information of the allocated antennas before actual data transmission. Therefore, this paper assumes the inclusion of allocated antenna information per subcarrier in L-SIG or VHT-SIG-A. Therefore, the overhead time of the conventional method is calculated by using binary phase shift keying (BPSK) and coding rate of $1 / 2$, as specified in IEEE 802.11. From this figure, the conventional method incurs more overhead as the number of STAs increases. For a massive antenna system with a great many spatial streams, the transmission efficiency is greatly degraded by the overhead increase. On the other hand, the proposed method performs to decide the receiving antenna comparing the received powers of the fixed preambles multiplied the proposed pre-coding, as shown in Section 3.2. Although the pre-coding value may change in accordance with the STA combination, the length of preambles with the proposed 
pre-coding does not change. In next generation wireless systems [3,4], discussions of increasing the number of streams beyond eight have been started. For example, when the number of streams is 16 and data length is $500 \mu \mathrm{s}$, we confirmed that the transmission efficiency of the system performance improves by about $24 \%$. Assuming more stream numbers, further improvement can be expected.

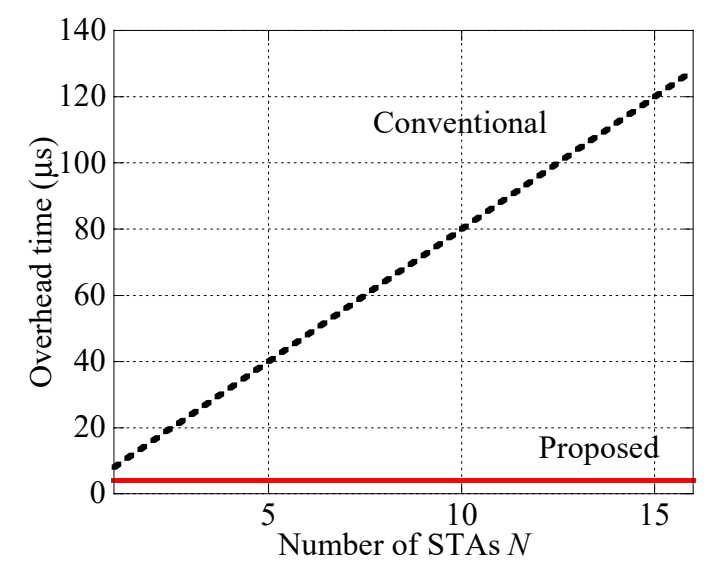

Figure 8. Overhead time vs. number of STAs.

Finally, we present the effectiveness of the proposed method. Figure 9 shows the CDF of the total channel capacity of the downlink MU-MIMO-OFDM transmission with a receiving antenna allocation by using the proposed method. Plots for five antenna decision variants are shown. The first uses the optimum antenna allocation without antenna decision error. The other four variants are the combinations of with/without the proposed pre-coding weight, which presents the receiving antenna decision method in Section 3.2, and the receiving antenna allocation with/without considering the antenna decision error in the limited CSI feedback sequence. It is clear that without the pre-coding, the proposed method has a greatly reduced performance because of the many antenna decision errors created by frequency selective fading. With the pre-coding, the channel capacity of the proposed method almost triples as the CDF value of $10 \%$. However, since the pre-coding cannot perfectly mitigate the received power, antenna decision error still occurs in response to the propagation environment. Moreover, the offsets from the optimal channel capacity are less than $20 \%$ at a CDF value of $50 \%$ because the decision error is improved. In detail, the proposal roughly quadruples the channel capacity at the $\mathrm{CDF}$ value of $10 \%$.

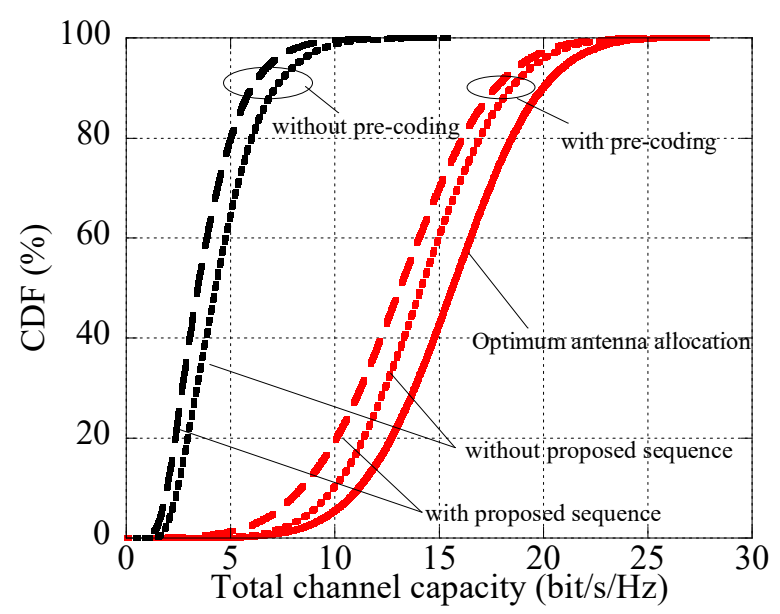

Figure 9. CDF of total channel capacities of the downlink MU-MIMO-OFDM transmission with/without the proposed pre-coding. 


\section{Conclusions}

In this paper, we proposed the receiving antenna allocation scheme for the downlink MU-MIMO-OFDM transmission; the AP simultaneously transmits data frames to a combination of allocated antennas per subcarrier at each STA. The first feature of the proposed scheme is the limited CSI feedback sequence; each STA feeds back to the AP just the channel response of the antenna with maximum norm if the spatial correlation value between receiving antennas is higher than a threshold. This sequence decreases the amount of CSI feedback and computational complexity while incurring only a small performance penalty in the downlink MU-MIMO-OFDM transmission.

The second feature of the proposed scheme is the receiving antenna decision method; the overhead to notify the allocated antenna information greatly decreases by the received power comparison.

To clarify the effectiveness of the proposed scheme, we evaluated it in a computer simulation using channel responses measured in an indoor environment. The results showed that the proposal matched the performance of the coordinated Tx-RX BD techniques. That is, the proposed scheme can maintain the channel capacity of the downlink MU-MIMO-OFDM transmission while greatly reducing the overhead and computer complexity.

Author Contributions: M.T., T.Y., and M.F. designed the proposed scheme; M.T. and M.F. performed the experiments and analyzed the results.

Funding: This research received no external funding.

Conflicts of Interest: The authors declare no conflict of interest.

\section{References}

1. Cisco Visual Networking Index: Global Mobile Traffic Forecast Update. Available online: https://www.cisco.com/c/en/us/solutions/collateral/service-provider/visual-networking-indexvni/mobile-white-paper-c11-520862.pdf (accessed on 26 July 2018).

2. Traffic and Market Data Report. Available online: https://www.ericsson.com/res/docs/2014/ericssonmobility-report-august-2014-interim.pdf (accessed on 26 July 2018).

3. Andrews, J.G.; Buzzi, S.; Choi, W.; Hanly, S.V.; Lozano, A.; Soong, A.C.; Zhang, J.C. What will 5G be? IEEE J. Sel. Areas Commun. 2014, 6, 1065-1082. [CrossRef]

4. Wang, C.X.; Haider, F.; Gao, X.; You, X.H.; Yang, Y.; Yuan, D.; Hepsaydir, E. Cellular architecture and key technologies for $5 \mathrm{G}$ wireless communication networks. IEEE Commun. Mag. 2014, 52, 122-130. [CrossRef]

5. Nakamura, T. 3GPP TR 36.913 - Requirements for further advancements for Evolved Universal Terrestrial Radio Access (EUTRA) LTE-Advanced. Available online: http:/ / www.tech-invite.com/3m36/tinv-3gpp-36913.html (accessed on 26 July 2018).

6. IEEE Computer Society LAN MAN Standards Committee. Wireless LAN Medium Access Control (MAC) and Physical Layer (PHY) Specifications. In IEEE Std. 802.11ac-2013; IEEE: Piscataway, NJ, US, 2013.

7. Larsson, E.L.; Edfors, O.; Tufvesson, F.; Marzetta, T.L. Massive MIMO for next generation wireless systems. IEEE Commun. Mag. 2014, 52, 185-195. [CrossRef]

8. Ngo, H.Q.; Larsson, E.G.; Marzetta, T.L. Energy and spectral efficiency of very large multiuser MIMO systems. IEEE Trans. Commun. 2013, 61, 1436-1449.

9. Spencer, Q.H.; Swindlehurst, A.L.; Haardt, M. Zero-forcing methods for downlink spatial multiplexing in multiuser MIMO channels. IEEE Trans. Signal Process. 2004, 52, 461-471. [CrossRef]

10. Kudo, R.; Takatori, Y.; Nishimori, K.; Ohta, A.; Kubota, S. A new user measure in block diagonalization for multiuser MIMO systems. IEICE Trans. Commun. 2009, E92-B, 3206-3218. [CrossRef]

11. Stankovic, V.; Haardt, M. Successive optimization Tomlinson-Harashima precoding (SO THP) for multi-user MIMO systems. In Proceedings of the IEEE International Conference on Acoustics, Speech, and Signal Processing, Philadelphia, PA, USA, 23 March 2005; Volume 3, pp. 1117-1120.

12. Peel, C.; Hochwald, B.; Swindlehurst, L. A vector-perturbation technique for near-capacity multiantenna multiuser communication-part I: Channel inversion and regularization. IEEE Trans. Commun. 2005, 53, 195-202. [CrossRef] 
13. Codreanu, M.; Tolli, A.; Juntii, M.; Latva-aho, M. Joint design of Tx-Rx Beamforers in MIMO downlink channel. IEEE Trans. Signal Process. 2007, 55, 4639-4655. [CrossRef]

14. Sadek, M.; Tarighat, A.; Sayed, A.H. Active antenna selection in multiuser MIMO communications. IEEE Trans. Signal Process. 2007, 55, 1498-1510. [CrossRef]

15. Zhang, X.; Lv, Z.; Wang, W. Performance analysis of multiuser diversity in MIMO systems with antenna selection. IEEE Trans. Wirel. Commun. 2008, 7, 15-21. [CrossRef]

16. Gore, D.A.; Paulraj, A.J. MIMO antenna subset selection with space-time coding. IEEE Trans. Signal Process. 2002, 50, 2580-2588. [CrossRef]

17. Shen, Z.; Chen, R.; Andrews, J.G.; Heath, R.W.; Evans B, L. Low complexity user selection algorithms for multiuser MIMO systems with block diagonalization. IEEE Trans. Signal Process. 2006, 54, 3658-3663. [CrossRef]

18. Ntontin, K.; Di Renzo, M.; Pérez-Neira, A.I.; Verikoukis, C. A low-complexity method for antenna selection in spatial modulation systems. IEEE Commun. Lett. 2013, 17, 2312-2315. [CrossRef]

19. Zhang, H.; Li, Y.; Stolpman, V.; Van Waes, N. A reduced CSI feedback approach for precoded MIMO-OFDM systems. IEEE Trans. Wirel. Commun. 2007, 6. [CrossRef]

20. Murakami, T.; Takatori, Y.; Mizoguchi, M.; Ujihara, K.; Maehara, F. Downlink multiuser MIMO-OFDM transmission using simple receive antenna selection. In Proceedings of the International Symposium on Antennas and Propagation, Ginowan, Japan, 24-28 October 2016; pp. 604-605.

21. Murakami, T.; Takatori, Y.; Mizoguchi, M.; Maehara, F. Antenna decision method for downlink multiuser MIMO systems with receive antenna allocation. In Proceedings of the 2015 IEEE International Symposium on Antennas and Propagation \& USNC/URSI National Radio Science Meeting, Vancouver, BC, Canada, 19-24 July 2015; pp. 304-305.

22. Keysight Technologies. Available online: https://literature.cdn.keysight.com/litweb/pdf/5989-7819EN. pdf?id=1364807 (accessed on 26 July 2018).

(C) 2018 by the authors. Licensee MDPI, Basel, Switzerland. This article is an open access article distributed under the terms and conditions of the Creative Commons Attribution (CC BY) license (http:/ / creativecommons.org/licenses/by/4.0/). 